\title{
Construcción y Evaluación Psicométrica de una Escala Breve de Vulnerabilidad Suicida
}

\section{Construction and Psychometric Evaluation of a Brief Scale of Suicidal Vulnerability}

\author{
Nicolás Sánchez-Álvarez ${ }^{1}$, Jaime de la Torre López ${ }^{2}$ y Lucía Pérez-Costillas ${ }^{3}$
}

\section{Resumen}

El suicidio es una de las principales causas de muerte evitable. La detección temprana de personas en riesgo es fundamental para reducir el número de fallecidos. En este estudio se ha desarrollado una escala para identificar el riesgo suicida, teniendo en cuenta no solo factores de riesgo sino también Fortalezas Personales del sujeto. El instrumento consta de 30 ítems y está orientado para su uso por parte de agentes sociales. Su diseño se testó en 95 usuarios de los servicios sociales mostrando una alta consistencia interna $(\alpha=.83)$. La validez del constructo se evaluó mediante la fiabilidad test-retest del instrumento, mostrando una alta fiabilidad $(\alpha=.72)$.

Palabras clave: suicidio, informantes clave, instrumento, construcción de pruebas psicológicas

\begin{abstract}
Suicide is one of the main causes of preventable death, so there is a great need to assess possible cases at risk. In this study, a scale has been developed to identify suicidal risk, taking into account not only risk factors but also personal strengths of the subject. The instrument consists of 30 items and is intended for use by social agents. Its design was tested on 95 users of social services showing a high internal consistency $(\alpha=.83)$. The validity of the construct was evaluated by the test-retest reliability of the instrument, showing a high reliability $(\alpha=.72)$.
\end{abstract}

Keywords: suicide, key informants, instrument, psychological testing

\footnotetext{
${ }^{1}$ PhD Psicología. Deusto Stress Research, Universidad de Deusto, 48007 Bilbao, España. Tel.: +34 676067492. Correo: nsa@deusto.es

${ }^{2} \mathrm{PhD}$ estudiante de posgrado Psicología. Universidad de Málaga, España. Campus Teatinos s/n 29071. Tel.: +34 680107437. Correo: jaime.delatorre@justalegria.org.

${ }^{3}$ PhD Psiquiatría. Unidad de Gestión Clínica de Salud Mental. Hospital Regional Universitario de Málaga, España. Instituto de investigación IBIMA. Málaga, España. Miembro del Grupo Andaluz de Investigación Psicosocial (GAP) (CTS-945). Av. De Carlos de Haya, s/n 29010. Tel.: +34 647915674. Correo: lpcostillas@ gmail.com

Revista Iberoamericana de Diagnóstico y Evaluación - e Avaliação Psicológica. RIDEP · №49 · Vol.4 · 23-35 · 2018

ISSN: 1135-3848 print /2183-6051online
} 


\section{Introducción}

La Organización Mundial de la Salud, OMS, define el suicidio como un problema mundial de primer orden, por ser una de las grandes causas prevenibles de muerte prematura. Esta institución promueve que gobiernos y sociedad civil desarrollen estrategias de prevención del suicidio, que incluyan programas y actividades comunitarias (World Health Organization, 2014). Desde 2008, el suicidio es la causa de muerte no natural más frecuente en España, por encima de los accidentes de tráfico. En 2015, el INE (Instituto Nacional de Estadística, 2017) informó de una tasa de suicidios en el país es de 7.76 por cada 100,000 habitantes. Las estrategias de prevención que han resultado más efectivas han sido la formación de profesionales de atención primaria y la detección de personas de alto riesgo (Ayuso-Mateos et al., 2012).

Existe la necesidad de contar con herramientas de fácil aplicación que permitan la detección temprana de personas con alto riesgo de suicidio (Anguiano, Mayer, Piven, \& Rosenstein, 2012; Stefansson, Nordström, \& Jokinen, 2012). Un reciente estudio de revisión sobre los instrumentos de evaluación del riesgo suicida muestra las carencias, destacando la complejidad de aplicación, ausencia de propiedades psicométricas adecuadas, baja discriminación, requerimiento de entrenamiento previo para su aplicación, entre otros (Rangel-Garzón, SuárezBeltrán, \& Escobar-Córdoba, 2015). Por ello, es imprescindible desarrollar escalas de fácil aplicación, breves, de fácil interpretación, y que puedan ser administradas por cualquier profesional de atención temprana sin necesidad de formación específica previa. Debido a las limitaciones de instrumentos de evaluación del riesgo suicida, el objetivo principal de esta investigación fue la construcción y validación de un instrumento preliminar para detectar personas en riesgo de suicidio, con unas características que no cumplen instrumentos de evaluación previos: utilidad, fácil distribución, fácil interpretación, y una alta fiabilidad.

La evaluación de la conducta suicida es una tarea compleja debido a la interacción de varios factores como son factores psicológicos, socioeconómicos, familiares, interpersonales y biológicos (Hawton \& van Heeringen, 2009). Para el ámbito socio-sanitario, el estudio del suicidio supone un reto enorme, sobre todo si se tiene en cuenta que el $83 \%$ de las personas que tuvieron un intento planificado y el $74.1 \%$ de las que cometieron un intento no planificado tienen un trastorno mental (Nock, Hwang, Sampson, \& Kessler, 2010). Otros factores de riesgo son el abuso de alcohol (Martinotti, Lupi, Santacroce, \& Di Giannantonio, 2014) y drogas (Yoshimasuet al.,2008), los antecedentes de abusos físicos y/o sexuales en la infancia (Joiner et al., 2007), el aislamiento social (Hall-Lande, Eisenberg, Christenson, \& Neumar-Sztainer, 2007), los trastornos mentales (Bernal et al., 2007; Nock et al., 2008), y las enfermedades somáticas incapacitantes (Crump, Sundquist, Sundquist, \& Winkleby, 2014). Haber tenido tentativas de suicidio previas (Beghi, Rosenbaum, Cerri, \& Cornaggia, 2013) y una historia familiar de suicidio y/o enfermedad mental también aumentan el riesgo suicida (Brent \& Melhem, 2008). Al mismo tiempo, existen algunos factores precipitantes que pueden aumentar el riesgo de suicidios. Los acontecimientos o circunstancias estresantes como el desempleo, dificultades económicas, las pérdidas de personas queridas, las discusiones con la familia o los amigos, la ruptura de relaciones, los problemas legales, laborales y financieros (McFeeters, Boyda, \& Siobhan, 2015; Pompili et al., 2011) pueden desencadenar conductas suicidas.

La investigación en el campo del suicidio se ha centrado prioritariamente en identificar los factores de riesgo (Marty, Segal, \& Coolidge, 2010), sin embargo se conoce menos sobre los factores protectores que puedan prevenir la conducta suicida (Arenas-Landgrave, Maqueo, \& Forns, 2011). Varios de los factores protectores encontrados dentro del nivel comunitario son la participación en actividades religiosas y comunitarias (Fässberg et al., 2012; Rasic, Robinson, Bolton, Bienvenu, \& Sareeen, 2011), y tener apoyo social (Kleiman \& Liu, 2013). Desde el reciente movimiento de la Psicología Positiva, se plantea que las personas presentan una serie de fortalezas psicológicas operacionalizadas en sistemas de creencias positivas, rasgos de personalidad diferenciales, estilos estables de pensamiento, y un conjunto de competencias 
sociales y emocionales, que ayudarían a afrontar el efecto negativo de los estresores cotidianos, fomentando el bienestar y ayudando a evitar la aparición de problemas psicológicos, incluido las ideaciones suicidas (Vázquez, Hervás, Rahona, \& Gómez, 2009). Por otro lado, a nivel intrapersonal destacan las habilidades personales que parecen incidir en la protección de aquellas variables envueltas en la tentativa de suicido tales como la gratitud (Kleiman, Adams, Kashdan, \& Riskind, 2013) o el optimismo (Rasmussen \& Wingate, 2011).

En este estudio se diseñó la elaboración de una escala de riesgo suicida que incluyera factores de riesgo y protectores, incluyendo habilidades personales. La escala debe ser una herramienta útil, rápida y de fácil uso para profesionales de Servicios Sociales Comunitarios y otros agentes sociales implicados en la detección temprana. Se evaluaron las propiedades psicométricas preliminares de la escala, al mismo tiempo, que se describen los factores positivos asociados a la protección del riesgo de suicidio.

\section{Método}

\section{Participantes}

En el estudio participaron 95 usuarios de los Servicios Sociales Comunitarios, de los cuáles el 64\% fueron mujeres, con un rango de edad de 19 a 75 años, y una media de 45 (DT=10). El 63.2\% afirmó tener pareja estable. En el nivel de estudios finalizados, un $57.9 \%$ terminó secundaria o formación profesional, un $32.6 \%$ educación primaria, el $6.3 \%$ no tiene estudios, y el $3.2 \%$ posee estudios universitarios. El $75 \%$ informa tener creencias religiosas. A nivel económico, el $76 \%$ indicó tener muchas dificultades para llegar a fin de mes. El screening inicial reveló que 13 participantes, un $14.3 \%$ del total de la muestra, han tenido algún intento de suicidio a lo largo de su vida. La selección de la muestra se realizó por un muestreo no probabilístico y accidental de los usuarios de un centro de Servicios Sociales de Málaga.

\section{Instrumentos}

Datos sociodemográficos. Se utilizó un cuestionario que evaluó: sexo, estado civil, nivel de estudios, actividad laboral, antecedentes personales y familiares de salud mental, sexualidad, consumo de alcohol, tabaco y otras drogas, abusos en la infancia, adolescencia y adultez, ingresos y pobreza subjetiva, y la historia de intentos de suicidio.

Lista de acontecimientos vitales estresantes (List of Threatening Experiences Questionnaire, LTE-Q; Brugha \& Cragg, 1990). El cuestionario selecciona los 12 sucesos vitales estresantes más potentes e importantes en la vida de las personas entrevistadas. Cada ítem corresponde a un suceso vital negativo. El participante responde si ha experimentado el suceso vital en el último año.

Escala de Ideación Suicida de Beck (Ideation Suicide Beck, ISB; Beck, Kovacs, \& Weissman, 1979). Escala hetero-administrada de 19 ítems aplicados en el contexto de una entrevista clínica semi-estructurada. Mide la intensidad de las actitudes, conductas y planes específicos para suicidarse. Cada ítem tiene 3 opciones de respuesta, con un rango de 0 a 2 . El rango de puntuación total es de entre 0 y 38 . Si la puntuación de los ítems (4) "Deseo de intentar suicidarse" y (5) "Intento pasivo de suicidarse", es " 0 ", se omiten las secciones siguientes y se suspende la entrevista, codificando como: "No aplicable". En caso contrario, se sigue aplicando (Beck et al., 1979).

Inventario de Depresión de Beck (Beck Depression Inventary, BDI; Beck, Rush, Shaw, \& Emery, 1979). Este instrumento cuenta con 21 ítems de formato auto-administrado que evalúa la severidad de los síntomas depresivos. Cada ítem se responde según la severidad de la sintomatología de 0 a 3 . El total de la puntuación de depresión se obtiene mediante la suma de los 21 ítems, obteniendo un rango de puntuación total de entre 0 y 63. Para este estudio se utilizó la versión adaptada al castellano (Sanz \& Vázquez, 1998).

Impulsividad Barratt (Barratt Impulsiveness Scale, BIS; Barratt, 1959). Para la evaluación de la impulsividad se utilizó la versión adaptada validada para población de habla castellana (Oquendo et al., 2001). Esta herramienta ofrece una puntuación total $\mathrm{y}$ tres sub-escalas: impulsividad cognitiva, impulsividad motora e impulsividad no planificada.

Entrevista Internacional Neuropsiquiatrica (Mini-International Neuropsychiatric Interview; MINI; Sheehan et al., 1998). Versión española 
5.0.0 (Bobes et al., 1997) usada para identificar presencia de enfermedad mental del eje I del DSM-IV, y trastorno de personalidad antisocial del eje II del DSM-IV.

Inventario de Fortalezas (Values in Action Inventory of Strengths, VIA-IS). Peterson \& Seligman, 2004), consta de 240 ítems con cinco posibilidades de respuesta: (5) "Muy Parecido a mí", (4) "Algo parecido a mí", (3) "Neutro", (2) "Algo diferente a mí", (1) "Nada parecido a mí". Distribuidos en seis dimensiones: sabiduría y conocimiento (50 ítems), coraje (40 ítems), humanidad y amor (30 ítems), justicia (30 ítems), templanza (40 ítems) y trascendencia (50 ítems). Mide 24 Fortalezas Personales que son: Creatividad, Curiosidad, Juicio, Aprendizaje, Perspectiva, Valentía, Perseverancia, Honestidad, Vitalidad, Amor, Amabilidad, Inteligencia Social, Trabajo en equipo, Equidad, Liderazgo, Perdón, Humildad, Prudencia, Autorregulación, Apreciación, Gratitud, Esperanza, Humor, y Espiritualidad. Los estudios de confiabilidad para la versión original (Peterson et al., 2004) y la versión al castellano (realizada en España por Vázquez \& Hervás, 2007), revelan valores de consistencia interna para cada uno de los factores superiores a .90 (Alfa de Cronbach).

\section{Procedimiento}

Los sujetos invitados a participar en este estudio fueron seleccionados entre las personas que acuden al Centro de Servicios Sociales de varios distritos de Málaga (sur de España) demandando ayuda. Tras explicar el estudio se solicitó su participación y la firma del consentimiento informado. Se propuso la colaboración a 179 sujetos y 95 aceptaron participar. Este estudio sigue los acuerdos pactados en la declaración de Helsinki.

Todos los participantes fueron evaluados con los instrumentos propuestos. La evaluación fue llevada a cabo por psicólogos voluntarios formados específicamente en la aplicación de las herramientas empleadas. La valoración de cada participante se realizó en una única sesión de una hora y media de duración. Los datos se incluyeron en una base de datos y fueron analizados con el software informático de análisis estadístico SPSS v19. Los análisis empleados fueron: para hallar las diferencias entre los individuos con intentos de suicidio y aquellos sin intentos la $T$ de Student para las variables continuas y el estadístico de contraste $X^{2}$ para las variables dicotómicas. Se utilizó el análisis factorial exploratorio para examinar las cargas factoriales, y el análisis factorial confirmatorio para comprobar el ajuste y la estructura factorial del instrumento. El ajuste del modelo fue testado mediante el indicador $X^{2}$ dividido por los grados de libertad $\left(X^{2} / g l<3\right)$, el índice de ajuste comparativo (Comparative Fit Index, $C F I>.90$ ), el índice de ajuste normalizado (Normed Fit Index, NFI > .90), y el índice del error de aproximación al cuadrado (Root Mean Square Error of Approximation index, RMSEA < .08). Para hallar la validez convergente y divergente se ha utilizado el índice de correlación de Pearson. Para la fiabilidad del instrumento se empleó el estadístico $\alpha$ de Cronbach. En los análisis de influencia indirecta se ha utilizado un sistema de ecuaciones estructurales con el software informático AMOS v19, con método de máxima verosimilitud.

Por último, se utilizó la técnica cualitativa Delphi para seleccionar y ordenar la importancia de los ítems que han resultado más significativos tras el análisis de datos. Un grupo de 15 expertos del grupo WORECA (Work Research Consortium for Suicide) evaluaron cada ítem de los seleccionados en el estudio estadístico con una puntuación de entre 0 y 4 según el nivel de severidad en relación con el riesgo suicida. Bajo las recomendaciones del grupo WORECA se agregaron las primeras 5 preguntas del instrumento, utilizadas para evaluar con rapidez un riesgo alto y la necesidad de derivación a servicios sanitarios especializados en casos de alto riesgo de suicidio.

\section{Resultados}

\section{Resultados comparativos}

Los análisis comparativos de las puntuaciones medias entre los participantes con intentos de suicidio y aquellos sin intentos, muestran diferencias significativas para los antecedentes familiares de depresión, antecedentes consumo de alcohol de familiares, número de acontecimientos vitales, ideación suicida, impulsividad motora, cognitiva y total (ver Tabla 1). También se hallan diferencias significativas entre los participantes 
Tabla 1. Resultados comparativos entre las personas con intento de suicidio y aquellos sin intentos

\begin{tabular}{|c|c|c|c|c|c|c|}
\hline Ítem & $\begin{array}{c}\text { Intento } \\
\text { Suicidio } \\
\end{array}$ & $\mathrm{N}$ & M & DT & $\mathrm{t}$ & $p$ \\
\hline \multirow{2}{*}{$\begin{array}{l}\text { Haber tenido un problema grave con algún } \\
\text { amigo cercano, vecino o familiar (LTE-Q) }\end{array}$} & No & 78 & .15 & .363 & -2.059 & .042 \\
\hline & Sí & 13 & .38 & .506 & & \\
\hline \multirow{2}{*}{$\begin{array}{l}\text { Haber tenido problemas con la policía o ha } \\
\text { comparecido ante un tribunal (LTE-Q) }\end{array}$} & No & 78 & .06 & .247 & -3.624 & .001 \\
\hline & Sí & 13 & .38 & .506 & & \\
\hline \multirow{2}{*}{ Ideación suicida (ISB) } & No & 78 & 3.55 & 1.918 & -1.998 & .049 \\
\hline & Sí & 13 & 7.94 & 6.543 & & \\
\hline \multirow{2}{*}{ Impulsividad cognitiva (BIS) } & No & 78 & 13.32 & 6.087 & -3.233 & .003 \\
\hline & Sí & 13 & 18.06 & 5.332 & & \\
\hline \multirow{2}{*}{ Impulsividad motora (BIS) } & No & 78 & 13.40 & 6.286 & -2.583 & .016 \\
\hline & Sí & 13 & 17.35 & 5.590 & & \\
\hline \multirow{2}{*}{ Impulsividad total (BIS) } & No & 78 & 42.81 & 13.426 & -2.473 & .015 \\
\hline & Sí & 13 & 51.82 & 14.518 & & \\
\hline \multirow[b]{2}{*}{ Ítem } & & \multicolumn{2}{|c|}{ Intento suicidio } & & & \\
\hline & & No & Sí & $X^{2}$ & & $p$ \\
\hline \multirow[t]{2}{*}{ Antecedentes familiares con depresión } & No & 55 & 4 & 4.831 & & .028 \\
\hline & $\mathrm{Si}$ & 28 & 8 & & & \\
\hline \multirow{2}{*}{ Abuso Infancia físico } & No & 73 & 10 & -2.811 & & .006 \\
\hline & Sí & 5 & 3 & & & \\
\hline \multirow{2}{*}{ Abuso Infancia abandono } & No & 75 & 10 & 4.493 & & .034 \\
\hline & Sí & 3 & 3 & & & \\
\hline \multirow{2}{*}{ Abuso Infancia emocional } & No & 70 & 10 & 6.964 & & .008 \\
\hline & Sí & 8 & 3 & & & \\
\hline \multirow{2}{*}{ Abuso Infancia perdida padre } & No & 74 & 10 & 6.128 & & .013 \\
\hline & Sí & 4 & 3 & & & \\
\hline \multirow{2}{*}{ Abuso Físico Adolescencia/Edad Adulta } & No & 73 & 10 & 7.841 & & .005 \\
\hline & Sí & 5 & 3 & & & \\
\hline \multirow{2}{*}{ Abuso Emocional Adolescencia/Edad Adulta } & No & 70 & 10 & 6.964 & & .008 \\
\hline & Sí & 8 & 3 & & & \\
\hline \multirow{2}{*}{ Historia previa tratamiento } & No & 54 & 3 & 6.909 & & .009 \\
\hline & Sí & 24 & 10 & & & \\
\hline \multirow{2}{*}{ Historia previa hospitalización } & No & 69 & 10 & 5.924 & & .015 \\
\hline & Sí & 9 & 3 & & & \\
\hline \multirow{2}{*}{ Tratamiento actual psiquiátrico } & No & 74 & 10 & 9.597 & & .002 \\
\hline & Sí & 4 & 3 & & & \\
\hline \multirow{2}{*}{ Tratamiento actual fármacos } & No & 55 & 3 & 5.299 & & .021 \\
\hline & Sí & 23 & 10 & & & \\
\hline \multirow{2}{*}{ Suicidio consumado entorno amigos } & No & 73 & 10 & 4.770 & & .029 \\
\hline & Sí & 5 & 3 & & & \\
\hline \multirow{2}{*}{ Depresión mayor (MINI) } & No & 47 & 3 & 5.360 & & .021 \\
\hline & Sí & 31 & 10 & & & \\
\hline \multirow{2}{*}{ Fobia Social (MINI) } & No & 73 & 10 & 4.770 & & .029 \\
\hline & Sí & 5 & 3 & & & \\
\hline Tort & No & 78 & 11 & 9.374 & & .002 \\
\hline Irastomo por abuso de arogas (MIINI) & Sí & 0 & 2 & & & \\
\hline Trastorno antisocial (MIND) & No & 78 & 12 & 4.637 & & .031 \\
\hline 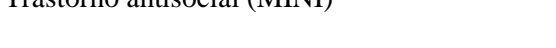 & Sí & 0 & 1 & & & \\
\hline
\end{tabular}


Tabla 2. Efectos indirectos significativos de las Fortalezas sobre el riesgo suicida vía impulsividad y depresión

\begin{tabular}{lcccccccc}
\hline Variable & Curiosidad & Aprendizaje & Perspectiva & Vitalidad & Prudencia & $\begin{array}{c}\text { Auto- } \\
\text { regulación }\end{array}$ & Esperanza & Espiritualidad \\
\hline & \multicolumn{7}{c}{ Vía Impulsividad } \\
$\begin{array}{l}\text { Riesgo } \\
\text { suicida }\end{array}$ & -.043 & -.029 & -.007 & -.056 & -.049 & -.021 & -.030 & -.028 \\
$\begin{array}{l}\text { Riesgo } \\
\text { suicida }\end{array}$ & -.003 & -.036 & - & -.048 & - & -.078 & -.042 & - \\
\hline
\end{tabular}

con intentos de suicidio y aquellos sin intentos en abuso físico y emocional en la infancia, adolescencia y edad adulta, historia psicopatológica, tratamiento actual psiquiátrico y farmacológico, suicidio consumado de un amigo, sintomatología depresiva, fobia social, abuso de drogas y trastorno antisocial.

\section{Resultados indirectos}

Se observan resultados significativos de efectos indirectos mediante análisis de mediación a través del uso de ecuaciones estructurales de los efectos de las fortalezas personales sobre el riesgo suicida. Las variables mediadoras fueron la impulsividad y la depresión. Como se puede observar en la Tabla 2, los efectos de las fortalezas mediadas por la impulsividad son la curiosidad, aprendizaje, perspectiva, vitalidad, prudencia, auto-regulación, esperanza y espiritualidad. También se observó un efecto indirecto a través de la depresión por parte de la curiosidad, aprendizaje, vitalidad, autoregulación, y esperanza.

\section{Construcción del instrumento}

De los resultados obtenidos se sustrajeron los ítems relativos a los antecedentes de suicidios personales y familiares, al igual que antecedentes psicopatológicos y abusos en la infancia y adolescencia, todas ellas variables tradicionalmente vinculadas a los intentos de suicidio. Al mismo tiempo, los resultados muestran que la conducta suicida también se relaciona con la sintomatología depresiva, trastornos fóbicos, abuso de drogas, impulsividad $\mathrm{y}$ acontecimientos vitales negativos. De forma indirecta, las fortalezas personales como curiosidad, amor por el aprendizaje, perspectiva, vitalidad, prudencia, auto-regulación, esperanza y espiritualidad se relacionan con la conducta suicida. Por lo que el instrumento para la evaluación del riesgo suicida se compone por ítems que evalúan dichas variables. Debido a la inclusión de los 5 primeros ítems por parte del comité de expertos de WORECA, la primera parte del instrumento es el denominado screening clínico para la rápida detección de casos graves. El resto de los ítems del instrumento tiene una baremación que incluye desde el ítem 6 hasta el 30, con una puntuación 1 para respuestas SI y 0 para respuestas NO.

\section{Ponderación de las puntuaciones}

Los resultados de las puntuaciones de la muestra con intento de suicidio previo y aquellos sujetos sin intento de suicidio en el instrumento de riesgo de suicidio se pueden observar en la Tabla 3. Los resultados comparativos muestran diferencias significativas en las puntuaciones del instrumento aportando validez discriminante.

Tabla 3. Medias y desviaciones típicas de las puntuaciones del instrumento del grupo con y sin intento de suicidio

\begin{tabular}{lcccc}
\hline Grupo & $\mathrm{N}$ & $\mathrm{M}$ & $\mathrm{DT}$ & $\mathrm{T}$ \\
\hline $\begin{array}{l}\text { Con intento de } \\
\text { suicidio }\end{array}$ & 78 & 11.07 & 3.602 & $4.362^{* * *}$ \\
$\begin{array}{l}\text { Sin intento de } \\
\text { suicidio }\end{array}$ & 13 & 15.35 & 3.936 & \\
\hline
\end{tabular}

Por otro lado, las puntuaciones descriptivas generales del instrumento muestran unos percentiles a raíz de los cuales se construyen la baremación de las puntuaciones. En la Tabla 4, se puede apreciar la distribución de las puntuaciones por el nivel de riesgo del instrumento.

\section{Análisis factorial}

Tanto el estadístico de adecuación muestral KMO (Kaiser, Meyer, \& Olkin test $=.503$ ) como la prueba de esfericidad de Barlett $\left(X^{2(300)}=435.655 ; p<.001\right)$ indican la pertinencia de 
Tabla 4. Estadísticos del instrumento y puntuaciones instrumento

\begin{tabular}{lccc}
\hline Percentiles & Puntuación & Rango de puntuaciones & Riesgo \\
\hline 25 & 8 & 0 a 8 & Riesgo suicidio bajo \\
50 & 11 & 9 a 11 & Riesgo suicidio leve \\
75 & 16 & 12 a 15 & Riesgo suicidio moderado \\
& $>16$ & Riesgo suicidio alto \\
\hline
\end{tabular}

Tabla 5. Estructura Factorial de la Escala Breve de Vulnerabilidad Suicida (EBVS), varianza explicada y fiabilidad por factor

\begin{tabular}{|c|c|c|c|c|c|c|c|c|c|c|}
\hline \multirow[b]{2}{*}{ Ítems } & \multicolumn{10}{|c|}{ Componentes } \\
\hline & $\begin{array}{l}\text { Factor } 1 \\
\text { (FP) }\end{array}$ & $\begin{array}{c}\text { Factor } 2 \\
\text { (IM) }\end{array}$ & $\begin{array}{c}\text { Factor } 3 \\
\text { (AB) }\end{array}$ & $\begin{array}{c}\text { Factor } 4 \\
\text { (AV) }\end{array}$ & $\begin{array}{l}\text { Factor } 5 \\
\text { (DE) }\end{array}$ & $\begin{array}{c}\text { Factor } 6 \\
\text { (AF) }\end{array}$ & $\begin{array}{c}\text { Factor } 7 \\
(\mathrm{PR})\end{array}$ & $\begin{array}{l}\text { Factor } 8 \\
\text { (SM) }\end{array}$ & $\begin{array}{c}\text { Factor } 9 \\
\text { (SE) }\end{array}$ & $\begin{array}{l}\text { Factor } 10 \\
\text { (RS) }\end{array}$ \\
\hline$\overline{\text { EBVS } 23}$ & .852 & & & & & & & & & \\
\hline EBVS 24 & .599 & & & & & & & & & \\
\hline EBVS 25 & .770 & & & & & & & & & \\
\hline EBVS 26 & .889 & & & & & & & & & \\
\hline EBVS 27 & .813 & & & & & & & & & \\
\hline EBVS 28 & .846 & & & & & & & & & \\
\hline EBVS 29 & .901 & & & & & & & & & \\
\hline EBVS 30 & .678 & & & & & & & & & \\
\hline EBVS 21 & & .736 & & & & & & & & \\
\hline EBVS 22 & & .894 & & & & & & & & \\
\hline EBVS 13 & & & .381 & & & & & & & \\
\hline EBVS 15 & & & .827 & & & & & & & \\
\hline EBVS 12 & & & & .642 & & & & & & \\
\hline EBVS 14 & & & & .634 & & & & & & \\
\hline EBVS 7 & & & & & .553 & & & & & \\
\hline EBVS 16 & & & & & .748 & & & & & \\
\hline EBVS 17 & & & & & .901 & & & & & \\
\hline EBVS 8 & & & & & & .779 & & & & \\
\hline EBVS 18 & & & & & & & .536 & & & \\
\hline EBVS 19 & & & & & & & .450 & & & \\
\hline EBVS 20 & & & & & & & .404 & & & \\
\hline EBVS 9 & & & & & & & & .529 & & \\
\hline EBVS 10 & & & & & & & & .735 & & \\
\hline EBVS 6 & & & & & & & & & .814 & \\
\hline EBVS 11 & & & & & & & & & .768 & \\
\hline EBVS 1 & & & & & & & & & & .811 \\
\hline EBVS 2 & & & & & & & & & & .434 \\
\hline EBVS 3 & & & & & & & & & & .604 \\
\hline EBVS 4 & & & & & & & & & & .874 \\
\hline EBVS 5 & & & & & & & & & & .700 \\
\hline $\begin{array}{l}\text { Varianza } \\
\text { explicada \% }\end{array}$ & $16.5 \%$ & $9.4 \%$ & $9 \%$ & $8.1 \%$ & $7.7 \%$ & $5.3 \%$ & $4.8 \%$ & $4.3 \%$ & $4 \%$ & $3.9 \%$ \\
\hline Alpha & .92 & .72 & .65 & .63 & .63 & - & .65 & .47 & .37 & .51 \\
\hline
\end{tabular}

Nota. FP=Fortalezas Personales; IM=Impulsividad; $\mathrm{AB}=\mathrm{Abuso}$ infancia/adolescencia; $\mathrm{AV}=$ Acontecimientos vitales estresantes; $\mathrm{DE}=$ Depresión; $\mathrm{AF}=\mathrm{Al}$ cohol familia; $\mathrm{PR}=$ Problemas corto plazo; $\mathrm{SM}=$ Salud mental; $\mathrm{SE}=$ Suicidio entorno; RS=Riego suicidio.

la realización de un análisis factorial. Se realiza en primera instancia un análisis factorial exploratorio con un método de extracción de componentes principales y con un método de rotación de normalización Varimax con Kaiser. Este análisis aporta una solución de diez factores con autovalores superiores a 1 que explican el $73 \%$ de la varianza. En segundo lugar se realizó un análisis factorial confirmatorio, cuyo índices de ajuste indican un ajuste de la estructura factorial apropiado $\quad\left(X^{2} / g l=1.47 ; \quad C F I=.95 ; \quad N F I=.93\right.$; $R M S E A=.07)$. En la Tabla 5 se puede observar la estructura factorial.

\section{Análisis de fiabilidad}

$\mathrm{El}$ factor 1 (Fortalezas Personales) con 8 ítems muestra una consistencia interna de .92. El factor 2 (Impulsividad) con 2 ítems muestra una consistencia interna de .72. El factor 3 (Abusos infancia/adolescencia) con 2 ítems muestra una consistencia interna de .65. El factor 4 (Acontecimientos vitales estresantes) con 2 ítems muestra una consistencia interna de .63. El factor 5 (Depresión) con 3 ítems muestra una consistencia interna de .63. El factor 6 (Alcohol en la familia) con 1 ítem no muestra consistencia interna. El factor 7 (Problemas a corto plazo) con 
3 ítems muestra una consistencia interna de .65. El factor 8 (Salud mental) con 2 ítems muestra una consistencia interna de .47. El factor 9 (Suicidio entorno) con 2 ítems muestra una consistencia interna de .37. El factor 10 (Riesgo suicidio) con 5 ítems muestra una consistencia interna de .51.

Para evaluar la fiabilidad test-retest de la escala se utilizó una sub-muestra de 61 usuarios de los servicios sociales que cumplimentaron de forma voluntaria el instrumento, y volvieron a cumplimentarlo por segunda vez a los 12 meses. Este tamaño sub-muestra para el estudio test-retest fue más reducido debido a dificultades para encontrar a los participantes. En la primera evaluación del instrumento, el alfa de Cronbach para la muestra fue $\alpha=.83$, y se mantuvo alta en la segunda evaluación con un $\alpha=.72$. Las correlaciones entre las medias de la prueba en ambas evaluaciones fue $r=.84$, lo que sugiere que la fiabilidad test-retest se encuentra dentro de los niveles adecuados.

\section{Discusión y Conclusiones}

En el análisis de los resultados destaca un alto porcentaje de participantes en el estudio que han presentado algún intento de suicidio a lo largo de su vida. Estos datos respaldan la sensibilidad de la población con dificultades socioeconómicas a sufrir un mayor riesgo de conductas suicidas y la necesidad de implementar estrategias encaminadas a incrementar la detección precoz. En relación a los datos socio-demográficos y los factores clásicos que influyen en el riesgo de suicidio, este estudio está en línea con los resultados obtenidos por investigaciones previas, destacando la importancia de variables como acontecimientos vitales estresantes (McFeeters et al., 2015), antecedentes familiares de depresión (Brent \& Melhem, 2008), alcoholismo (Martinotti et al., 2014), impulsividad (Dvorak, Lamis, \& Malone, 2013), abusos en infancia, adolescencia o edad adulta (Joiner et al., 2007), psicopatología previa o actual (Nock et al., 2008), depresión, fobia social (Davidson, Wingate, Grant, Judah, \& Mills, 2011) o consumo de drogas (Yoshimasu et al., 2008).

Además de existir una serie de factores de riesgo, existen factores protectores que disminuyen o previenen el riesgo suicida, permitiendo que personas ante las mismas situaciones estresantes puedan evaluar las situaciones como menos estresantes (ArenasLandgrave et al., 2011). Dentro de estos factores protectores se encuentran los recursos personales. En este estudio se han evaluado las fortalezas personales de los sujetos participantes, y algunas se han mostrado como variables influyentes en el riesgo suicida de forma indirecta, mostrando este efecto a través de la depresión (Han, Kim, Lee, Pistulka, \& Kim, 2007) y la impulsividad (Gvion \& Apter, 2011). Las fortalezas que han tenido una influencia significativa han sido: Curiosidad (Denneson, Smolenski, Bush, \& Dobscha, 2017), Pasión por aprender, Perspectiva, Vitalidad, Prudencia, Auto-regulación, Esperanza y Espiritualidad (Taliaferro, Rienzo, Pigg, Miller, \& Dodd, 2009). La teoría del enfoque de prevención de los recursos personales parte de la idea del papel protector de ciertas habilidades que al desempeñarlas o hacer uso de ellas provocan en el sujeto la aparición de un estado emocional positivo (Gvion \& Apter, 2011), siendo contrario a un estado emocional negativo relacionado con los pensamientos suicidas (Vázquez et al., 2009). Estos resultados son sumamente importantes ya que permite diseñar un instrumento que proporcione información adicional de los niveles de protección de las personas evaluadas frente a situaciones que puedan llevar a conductas suicidas.

Este trabajo presenta un instrumento piloto de detección de población en riesgo suicida cumpliendo con la premisa de fácil aplicación. Ha sido fruto de las evaluaciones realizadas a una muestra significativa de población en situación de riesgo, y validado a través del juicio de expertos que han consensuado la relevancia de los ítems de la escala (Rangel-Garzón et al., 2015). Los índices de consistencia interna son correctos pese a que algunos factores tienen unos índices bajos debido a la cantidad de elementos que incluyen cada factor. En su construcción, se han empleado variables que muestran diferencias entre personas que han tenido intentos de suicidio con otras que no dentro del mismo grupo de población vulnerable. Es necesario continuar este trabajo con un nuevo diseño de tipo longitudinal para comprobar su efectividad en la detección de población en riesgo de suicidio para incorporarlo 
como herramienta de trabajo de agentes comunitarios en contacto con población de riesgo. Se plantean como futuras líneas de investigación la validación del instrumento en una población más amplia, al mismo tiempo que se consensuan los resultados en distintos sectores poblacionales.

\section{Financiación}

Área de Derechos Sociales del Ayuntamiento de Málaga (España).

\section{Agradecimientos}

Ayuntamiento de Málaga, Área de Derechos Sociales, Centro de Servicios Sociales del Distrito Bailén Miraflores, Asociación Justalegría, Asociación Internacional del Teléfono de la Esperanza.

\section{Referencias}

Anguiano, L., Mayer, D. K., Piven, M. L., \& Rosenstein, D. (2012). A literature review of suicide in cancer patients. Cancer Nursing, 35(4), E14-E26. doi:10.1097/NCC.0b013e31822fc76c

Arenas-Landgrave, P., Maqueo, E. L. G., \& Forns, M. (2012). Indicadores diferenciales de personalidad frente al riesgo de suicidio en adolescentes. Differential personality indicators in the face of suicidal risk in adolescents. Revista Iberoamericana de Diagnóstico y Evaluación - e Avaliação Psicológica, 33(1), 51-74.

Ayuso-Mateos, J. L., Baca-García, E., Bobes, J., Giner, J., Giner, L., Pérez, V., ...\& RECOMS, G. (2012). Recomendaciones preventivas y manejo del comportamiento suicida en España. Revista de Psiquiatría y Salud Mental, 5(1), 8-23. doi:org/10.1016/j.rpsm.2012.01.001

Barratt, E. S. (1959). Anxiety and impulsiveness related to psychomotor efficiency. Perceptual and Motor Skills, 9(3), 191-198. doi:10.2466/pms.1959.9.3.191

Beck, A. T., Kovacs, M., \& Weissman, A. (1979). Assessment of suicidal intention: The Scale for Suicide Ideation. Journal of Consulting And Clinical Psychology, 47(2), 343. doi:org/10.1037/0022-006X.47.2.343
Beck, A. T., Rush, A. J., Shaw, B. F., \& Emery, G. (1979). Cognitive Therapy of Depression. New York: Guilford Press.

Bernal, M., Haro, J. M., Bernert, S., Brugha, T., de Graaf, R., Bruffaerts, R., ...\& Torres, J. V. (2007). Risk factors for suicidality in Europe: Results from the ESEMED study. Journal of Affective Disorders, 101(1), 27-34. doi:org/10.1016/j.jad.2006.09.018

Bobes, J., Gutierrez, M., Palao, D., Ferrando, L., Gibert-Rahola, J., \& Lecrubier, Y. (1997). Validez del MINI (Mini International Neuropsychiatric Interview) en tres centros de AP en España. Psiquiatric Biology, 4(2), 79.

Beghi, M., Rosenbaum, J. F., Cerri, C., \& Cornaggia, C. M. (2013). Risk factors for fatal and nonfatal repetition of suicide attempts: A literature review. Neuropsychiatric Disease and Treatment 9(1): 1725-1736. doi:10.2147/NDT.S40213.

Brent, D. A., \& Melhem, N. (2008). Familial transmission of suicidal behavior. Psychiatric Clinics of North America, 31(2), 157-177. doi:10.1016/j.psc.2008.02.001

Brugha, T. S., \& Cragg, D. (1990). The list of threatening experiences: The reliability and validity of a brief life events questionnaire. Acta Psychiatrica Scandinavica, 82(1), 77-81. doi:10.1111/j.1600-0447.1990.tb01360.x

Crump, C., Sundquist, K., Sundquist, J., \& Winkleby, M. A. (2014). Sociodemographic, psychiatric and somatic risk factors for suicide: A Swedish national cohort study. Psychological Medicine, 44(02), 279-289. Doi:10.1017/S0033291713000810

Davidson, C. L., Wingate, L. R., Grant, D. M., Judah, M. R., \& Mills, A. C. (2011). Interpersonal suicide risk and ideation: The influence of depression and social anxiety. Journal of Social and Clinical Psychology, 30(8), 842-855. doi:10.1521/jscp.2011.30.8.842

Denneson, L. M., Smolenski, D. J., Bush, N. E., \& Dobscha, S. K. (2017). Curiosity improves coping efficacy and reduces suicidal ideation severity among military veterans at risk for suicide. Psychiatry Research, 249, 125-131. doi:10.1016/j.psychres.2017.01.018

Dvorak, R. D., Lamis, D. A., \& Malone, P. S. (2013). Alcohol use, depressive symptoms, and impulsivity as risk factors for suicide 
proneness among college students. Journal of Affective Disorders, 149(1), 326-334. doi:10.1016/j.jad.2013.01.046

Fässberg, M. M., Orden, K. A. V., Duberstein, P., Erlangsen, A., Lapierre, S., Bodner, E., ...\& Waern, M. (2012). A systematic review of social factors and suicidal behavior in older adulthood. International Journal of Environmental Research And Public Health, 9(3), 722-745. doi:10.3390/ijerph9030722

Gvion, Y., \& Apter, A. (2011). Aggression, impulsivity, and suicide behavior: A review of the literature. Archives of Suicide Research, 15(2), 93-112. doi:10.1080/13811118.2011.565265

Han, H. R., Kim, M., Lee, H. B., Pistulka, G., \& Kim, K. B. (2007). Correlates of depression in the Korean American elderly: Focusing on personal resources of social support. Journal of Cross-Cultural Gerontology, 22(1), 115127. doi:10.1007/s10823-006-9022-2

Hall-Lande, J. A., Eisenberg, M. E., Christenson, S. L., \& Neumark-Sztainer, D. (2007). Social isolation, psychological health, and protective factors in adolescence. Adolescence, 42(166), 265.

Hawton, K., \& van Heeringen, K. (2009). Suicide. The Lancet, 373(9672), 1372-1381. doi:10.1016/S0140-6736(09)60372-X

Instituto Nacional de Estadística (2014). Defunciones según la causa de Muerte en España en el año 2012. Instituto Nacional de Estadística, 1-14. Retrieved from http://www.ine.es/prensa/np830.pdf

Joiner, T. E., Sachs-Ericsson, N. J., Wingate, L. R., Brown, J. S., Anestis, M. D., \& Selby, E. A. (2007). Childhood physical and sexual abuse and lifetime number of suicide attempts: A persistent and theoretically important relationship. Behaviour Research and Therapy, 45(3), 539-547. doi:10.1016/j.brat.2006.04.007

Kleiman, E. M., Adams, L. M., Kashdan, T. B., \& Riskind, J. H. (2013). Gratitude and grit indirectly reduce risk of suicidal ideations by enhancing meaning in life: Evidence for a mediated moderation model. Journal of Research in Personality, 47(5), 539546.Doi:10.1016/j.jrp.2013.04.007

Kleiman, E. M., \& Liu, R. T. (2013). Social support as a protective factor in suicide:
Findings from two nationally representative samples. Journal of Affective Disorders, 150(2), 540-545. doi:0.1016/j.jad.2013.01.033

Martinotti, G., Lupi, M., Santacroce, R., \& Di Giannantonio, M. (2014). Alcohol use disorders and suicidal behaviour: A clinical review of studies in developed and developing countries. Research and Advances in Psychiatry, 1(1), 20-31.

Marty, M. A., Segal, D. L., \& Coolidge, F. L. (2010). Relationships among dispositional coping strategies, suicidal ideation, and protective factors against suicide in older adults. Aging and Mental Health, 14(8), 10151023.

McFeeters, D., Boyda, D., \& Siobhan, O. (2015). Patterns of stressful life events: Distinguishing suicide ideators from suicide attempters. Journal of Affective Disorders, 175, 192-198. doi:10.1080/13607863.2010.501068

Nock, M. K., Borges, G., Bromet, E. J., Alonso, J., Angermeyer, M., Beautrais, A., ...\& De Graaf, R. (2008). Cross-national prevalence and risk factors for suicidal ideation, plans and attempts. The British Journal of Psychiatry, 192(2), 98-105. doi:10.1192/bjp.bp.107.040113

Nock, M. K., Hwang, I., Sampson, N. A., \& Kessler, R. C. (2010). Mental disorders, comorbidity and suicidal behavior: Results from the National Comorbidity Survey Replication. Molecular Psychiatry, 15(8), 868-876. doi:10.1038/mp.2009.29

Peterson, C., \& Seligman, M. E. (2004). Character strengths and virtues: A handbook and classification (Vol. 1). Oxford University Press.

Pompili, M., Innamorati, M., Szanto, K., Di Vittorio, C., Conwell, Y., Lester, D., ...\& Amore, M. (2011). Life events as precipitants of suicide attempts among first-time suicide attempters, repeaters, and non-attempters. Psychiatry Research, 186(2), 300-305. doi:10.1016/j.psychres.2010.09.003

Oquendo, M. A., Baca-García, E., Graver, R., Morales, M., Montalvan, V., \& Mann, J. J. (2001). Spanish adaptation of the Barratt impulsiveness scale (BIS-11). The European Journal of Psychiatry, 15(3), 147-155. 
Rangel-Garzón, C. X., Suárez-Beltrán, M. F., \& Escobar-Córdoba, F. (2015). Escalas de evaluación de riesgo suicida en atención primaria. Revista de la Facultad de Medicina, 63(4), 707-716. doi:10.15446/revfacmed.v63.n4.50849

Rasmussen, K. A., \& Wingate, L. R. (2011). The Role of optimism in the interpersonalpsychological theory of suicidal behavior. Suicide and Life-Threatening Behavior, 41(2), 137-148. doi:10.1111/j.1943-278X.2011.00022.x

Rasic, D., Robinson, J. A., Bolton, J., Bienvenu, O. J., \& Sareen, J. (2011). Longitudinal relationships of religious worship attendance and spirituality with major depression, anxiety disorders, and suicidal ideation and attempts: Findings from the Baltimore epidemiologic catchment area study. Journal of Psychiatric Research, 45(6), 848-854. doi:10.1016/j.jpsychires.2010.11.014

Sanz, J., \& Vázquez, C. (1998). Fiabilidad, validez y datos normativos del inventario para la depresión de Beck. Psicothema, 10(2), 303318. doi:7467-13170-1

Sheehan, D. V., Lecrubier, Y., Sheehan, K. H., Amorim, P., Janavs, J., Weiller, E., ...\& Dunbar, G. C. (1998). The Mini-International Neuropsychiatric Interview (MINI): The development and validation of a structured diagnostic psychiatric interview for DSM-IV and ICD-10. Journal of Clinical Psychiatry, (59), 22-33.

Stefansson, J., Nordström, P., \& Jokinen, J. (2012). Suicide Intent Scale in the prediction of suicide. Journal of Affective Disorders, 136(1), 167-171. doi:10.1016/j.jad.2010.11.016

Taliaferro, L. a., Rienzo, B. a., Pigg, R. M., Miller, M. D., \& Dodd, V. J. (2009). Spiritual well-being and suicidal ideation among college students. Journal of American College Health, 58(1), 83-90. doi:10.3200/JACH.58.1.83-90

Vázquez, C., \& Hervás, G. (2007). Adaptación española del Cuestionario VIA de Fortalezas. Retrieved from http://www. authentichappiness. sas. upenn. edu/Default. aspx.

Vázquez, C., Hervás, G., Rahona, J. J., \& Gómez, D. (2009). Psychological well-being and health. Contributions of positive psychology.
Anuario de Psicologia Clinica y Salud, 5, 1527.

World Health Organization (2014). Preventing suicide, 143(7), 609-610. https://doi.org/ISBN: 9789241564779

Yoshimasu, K., Kiyohara, C., Miyashita, K., et al. (2008). Suicidal risk factors and completed suicide: Meta-analyses based on psychological autopsy studies. Environmental health and preventive medicine, 13(5), 243256. doi:10.1007/s12199-008-0037-x 
Anexo I

\section{Instrumento piloto de identificación de población en riesgo ante la conducta suicida para agentes comunitarios}

A continuación, encontrará algunas afirmaciones sobre sus acontecimientos vitales, personales y familiares. Lea atentamente cada frase y marque la opción que más se aproxime a su situación.

\begin{tabular}{|c|c|c|}
\hline & SI & NO \\
\hline 1. En el último mes, ¿ha deseado estar muerto(a) o poder dormirse y no despertar? & & \\
\hline 2. En el último mes, ¿ha tenido realmente la idea de suicidarse? & & \\
\hline 3. ¿Ha pensado en cómo llevaría esto a cabo? & & \\
\hline 4. ¿Ha tenido estas ideas y en cierto grado la intención de llevarlas a cabo? & & \\
\hline $\begin{array}{l}\text { 5. ¿Ha comenzado a elaborar o ha elaborado los detalles sobre cómo suicidarse? ¿Tiene intenciones de } \\
\text { llevarlo a cabo? }\end{array}$ & & \\
\hline 6. En su familia, ¿ha habido algún intento de suicidio? & & \\
\hline 7. En su familia, ¿ha habido algún caso de depresión mayor (más de tres meses o medicación)? & & \\
\hline 8. En su familia, ¿ha habido algún caso de alcoholismo? & & \\
\hline 9. Actualmente, ¿tiene algún tratamiento? & & \\
\hline 10. A lo largo de su vida, ¿ha estado hospitalizado en la unidad de salud mental? & & \\
\hline 11. En su entorno de amigos, ¿ha habido algún suicidio consumado? & & \\
\hline 12. En su infancia, ¿sufrió el abandono de alguno de sus padres? & & \\
\hline 13. En su infancia, ¿sufrió la pérdida de su padre? & & \\
\hline 14. A lo largo de su vida, ¿ha sufrido abuso físico? & & \\
\hline 15. A lo largo de su vida, ¿ha sufrido abuso emocional? & & \\
\hline $\begin{array}{l}\text { 16. ¿En las últimas dos semanas se ha sentido deprimido o decaído la mayor parte del día, casi todos } \\
\text { los días? }\end{array}$ & & \\
\hline $\begin{array}{l}\text { 17. ¿En las últimas dos semanas, ha perdido el interés en la mayoría de las cosas o ha disfrutado menos } \\
\text { de las cosas que usualmente le agradaban? }\end{array}$ & & \\
\hline 18. En los últimos 6 meses, ¿ha tenido un problema grave con algún amigo cercano, vecino o familiar? & & \\
\hline 19. En los últimos 6 meses, ¿ha sufrido usted mismo una enfermedad, lesión o agresión grave? & & \\
\hline 20. En los últimos 6 meses, ¿ha tenido problemas con la policía o ha comparecido ante un tribunal? & & \\
\hline $\begin{array}{l}\text { 21. Mis pensamientos pueden tener muchas velocidad (tengo pensamientos que van muy rápidos en mi } \\
\text { mente) }\end{array}$ & & \\
\hline 22. Soy una persona con autocontrol & & \\
\hline $\begin{array}{l}\text { 23.Piense en situaciones reales en las cuales usted ha tenido la oportunidad de explorar algo nuevo o } \\
\text { de hacer algo diferente. ¿Muestra CURIOSIDAD o INTERÉS en estas situaciones? }\end{array}$ & & \\
\hline $\begin{array}{l}\text { 24.Piense en situaciones reales en las cuales usted ha tenido la oportunidad de aprender más sobre un } \\
\text { tema, dentro o fuera de la escuela/universidad. ¿Muestra PASIÓN POR APRENDER en estas } \\
\text { situaciones? }\end{array}$ & & \\
\hline $\begin{array}{l}\text { 25.Piense en situaciones reales en las cuales usted ha tenido la oportunidad de aconsejar a otra persona } \\
\text { que lo necesitaba. ¿En estas situaciones usted vio las cosas con PERSPECTIVA o SABIDURÍA? }\end{array}$ & & \\
\hline $\begin{array}{l}\text { 26.Piense en su vida cotidiana. ¿Muestra usted VITALIDAD o ENTUSIASMO cuando es posible } \\
\text { hacerlo? }\end{array}$ & & \\
\hline $\begin{array}{l}\text { 27.Piense en situaciones reales en las cuales usted ha sido tentado de hacer algo de lo que podría } \\
\text { haberse arrepentido después. ¿Muestra usted PRUDENCIA, DISCRECIÓN, o CUIDADO en estas } \\
\text { situaciones? }\end{array}$ & & \\
\hline $\begin{array}{l}\text { 28.Piense en situaciones reales en las cuales usted ha experimentado antojos, deseos, impulsos, o } \\
\text { emociones que usted deseaba controlar. ¿Muestra usted AUTO-CONTROL o AUTO- } \\
\text { REGULACIÓN en estas situaciones? }\end{array}$ & & \\
\hline $\begin{array}{l}\text { 29.Piense en situaciones reales en las cuales usted ha experimentado un fracaso o un contratiempo. } \\
\text { ¿Muestra usted ESPERANZA u OPTIMISMO en estas situaciones? }\end{array}$ & & \\
\hline $\begin{array}{l}\text { 30.Piense en su vida cotidiana. ¿Muestra usted RELIGIOSIDAD o ESPIRITUALIDAD cuando es } \\
\text { posible hacerlo? }\end{array}$ & & \\
\hline
\end{tabular}




\section{Instrucciones para la corrección}

Este instrumento cuenta con dos escalas, una primera escala de carácter clínico, ítems 1 a 5, y una segunda escala diseñada a medida para usuarios de servicios sociales.

\section{Items 1 a 5 (Screening Clínico)}

\section{Items 1 y 2:}

2 puntos $\rightarrow$ Riesgo alto

1 punto $\rightarrow$ Sigo evaluando, ítems 3, 4 y 5

0 puntos $\rightarrow$ paso a ítem 6

\section{Items 3, 4, 5:}

1,2 o 3 puntos $\rightarrow$ Riesgo alto

0 puntos $\rightarrow$ paso a ítem 6

\section{Items 6 a 30}

Esta escala evalúa el riesgo de suicidio mediante la suma de todos sus ítems. Sume un punto por cada "Sí" entre las preguntas 6 y 22. Sume un punto por cada "No" entre las preguntas 23 y 30. Compare el resultado con la tabla que se muestra a continuación y determine el riesgo de suicidio del individuo evaluado.

\begin{tabular}{ll}
\hline Riesgo suicidio bajo & 0 a 8 \\
Riesgo suicidio leve & 9 a 11 \\
Riesgo suicidio moderado & 12 a 15 \\
Riesgo suicidio Alto & $>16$ \\
\hline
\end{tabular}

